\title{
ACCURACY ASSESSMENT OF DIFFERENT ERROR ADJUSTMENT METHODS IN CLOSED TRAVERSE NETWORKS; STUDYING THE IMPACT OF DIFFERENT OBSERVATION ERROR SETUPS IN DIFFERENT GEOMETRICAL CONFIGURATIONS
}

\author{
H. Amini ${ }^{1, *}, \mathrm{~S}$. Mehrdad $^{1}$ \\ ${ }^{1}$ Depart. of Geomatics Eng., Faculty of Civil Engineering \& Transportation, U. of Isfahan, Isfahan, Iran - (hosseinamini1994, \\ sinamhr)@gmail.com
}

Commission IV, WG IV/8

KEY WORDS: Surveying Network Adjustment, Least Squares, Transit, Bowditch, Doubly-braced Quadrilateral, Error Propagation

\begin{abstract}
:
Similar to all infrastructural works, in order to directly prepare a map, one must act in a whole-to-part way. First, a framework containing certain coordinated points which can be used as base points for subsidiary measurements must be provided, relying on which various surveying tasks can be carried out. By means of solutions, the observation errors in determining the stable points should be propagated between all the observations. In the past, classical methods have been used due to the lack of facilities that can perform numerous calculations in a short time. In this project, we analyzed the accuracy of traditional or classical methods of error propagation in comparison with the Least Squares using simulated observational data with different accuracies. Then, with the output of different methods, the error ellipses are drawn, according to which, these outputs are compared with each other in terms of accuracy. Bowditch method resembled the results of the Least Squares in many cases while Transit method generally showed poorer accuracy and a dependence on the direction of the adjustments. Bowditch method was found to be getting closer to or even more accurate than the Lest Squares when increasing. The whole methods reached a better performance when the accuracy of angular and longitudinal observations were of the same order. Moreover, the Doubly-braced Quadrilateral and the Least Squares with constant weight were of equal accuracies, however, the accuracy of the true-weighted error propagation method outperformed the other methods.
\end{abstract}

\section{INTRODUCTION}

One of the common procedures for providing the right framework is to create surveying networks which are a set of consecutive points or stations that are coordinated by successive measurements of lengths and angles, and form a network of horizontal canvas points. The main purpose of the survey is to fix specified points to help link the subsidiary points together (Gutti, 2019; Chrzanowski, 1965). Moreover, the accuracy of the survey points should be far greater than the accuracy of the subsidiary points. Depending on the geometry of the points, the survey networks are divided into two categories: open and closed traverse networks. In open traverses, the start and end points of the traverse are two separate points, while in closed ones, the start and end points coincide.

In each traverse, depending on the method and facilities available, it is necessary to measure lengths and/or angles. Then, various adjustment methods need to be carried out to propagate the errors of the observations on the traverse points. In fact, in these methods, the goal is to find the coordinates of the points of the traverse network with the least possible errors.

Here, we evaluate and compare error adjustment methods in closed traverse networks and examine their efficiency under different conditions in terms of geometry, and the accuracy of the input data. In the next section, error adjustment methods, simulated observations, and evaluation methods are introduced respectively. And in the final section, the results are reported.

\section{ERROR ADJUSTMENT METHODS}

The measurements of a closed traverse network are not always convergent. Therefore, the responsibility of the adjustment methods is to propagate the errors between the observations, so that the observations become homogeneous and create a convergent shape. To do this, methods have been developed from the past to the present that have evolved over time with the advancements of mathematics as well as the possibility of highvolume computing in a short time by computers. In this section, some common error adjustment methods would be briefly introduced. Here, we introduced the local closing error in closed traverse networks prior to the Bowditch and Transit methods which are committed to adjust this error.

\subsection{Local Closing Error in Closed Traverse Networks}

In closed traverse networks, if the adjusted azimuths of the angles are $\mathrm{G}_{1}, \mathrm{G}_{2}$, etc., and the lengths of the sides are $\mathrm{L}_{1}, \mathrm{~L}_{2}$, etc., respectively (Figure 1), and we assume that the coordinates of the starting point (A) are known, we will have:

$$
\begin{aligned}
& \sum_{i}^{n} \Delta E_{i}=0 \\
& \sum_{i}^{n} \Delta N_{i}=0
\end{aligned}
$$

\footnotetext{
* Corresponding author
} 
where

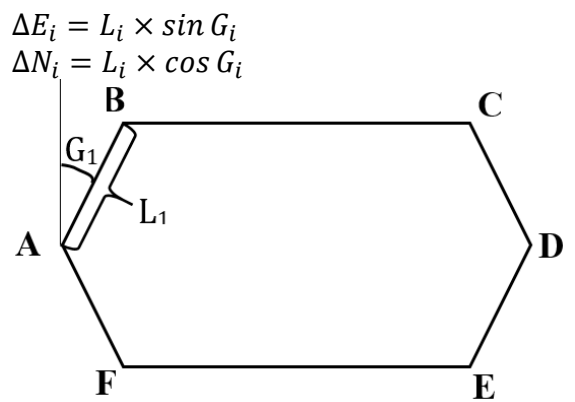

Figure 1. A closed traverse network. The azimuth angle $\left(\mathrm{G}_{1}\right)$ and the length $\left(\mathrm{L}_{1}\right)$ of $\mathrm{AB}$ are specified.

The equation 1 and equation 2 are called the polygonal condition in the closed traverse network. The intervention of measurement errors prevents the establishment of the polygonal condition. The traverse closing error is obtained from the difference between the two sides of the above equation as follows:

$$
\begin{aligned}
& \mathrm{F}_{E}=\sum_{i}^{n} \Delta E_{i} \\
& \mathrm{~F}_{N}=\sum_{i}^{n} \Delta N_{i} \\
& \mathrm{D}=\sqrt{\mathbf{F}_{E}^{2}+\mathbf{F}_{N}^{2}} \\
& G=\tan ^{-1}\left(\frac{\mathbf{F}_{E}}{\mathrm{~F}_{N}}\right)
\end{aligned}
$$

The vector with azimuth $\mathrm{G}$ and length $\mathrm{D}$ is called the error vector. This vector is the result of both longitudinal measurement errors and angular measurement errors. The length of this vector is called the local closing error.

\subsection{Bowditch Method}

In this method, the displacement of each point is parallel to the error vector and is proportional to the total distances of the points from the origin of the traverse (GTM, 1938). Therefore, the adjustment applied to each of the E (Easting) and N (Northing) coordinates of the points is as follows.

Adjustment of $\mathrm{E}$ for the ith vertex:

$$
\mathrm{CE}_{i}=\frac{-\mathrm{F}_{E}}{\sum_{i}^{n} \mathrm{~L}_{i}}\left(\mathrm{~L}_{1}+\mathrm{L}_{2}+\ldots+\mathrm{L}_{i}\right)
$$

Adjustment of $\mathrm{N}$ for the ith vertex:

$$
\mathrm{CN}_{i}=\frac{-\mathrm{F}_{N}}{\sum_{i}^{n} \mathrm{~L}_{i}}\left(\mathrm{~L}_{1}+\mathrm{L}_{2}+\ldots+\mathrm{L}_{i}\right)
$$

\subsection{Transit Method}

In this method, the displacement of the points is parallel to the error vector and is proportional to the absolute values of $\Delta$ Es and $\Delta$ Ns (Kimmick, 1949). Therefore, the adjustment applied to each of the relative coordinates is as follows.

$$
\begin{aligned}
& \mathrm{CE}_{i}=\frac{-\mathrm{F}_{E}}{\sum_{i}^{n} \Delta E_{i}} \times\left|\Delta E_{i}\right| \\
& \mathrm{CN}_{i}=\frac{-\mathrm{F}_{N}}{\sum_{i}^{n} \Delta N_{i}} \times\left|\Delta N_{i}\right|
\end{aligned}
$$

\subsection{Error Adjustment for Doubly-braced Quadrilateral}

In this method only angular observations have been performed. This method is usually used in areas where the width of the area is short compared to its length. In this method, four points including $\mathrm{A}, \mathrm{B}, \mathrm{C}$ and $\mathrm{D}$, which form the vertices of a quadrilateral, are stationed (Thornton-Smith, 1964). At each station, three other points are sighted, and a total of eight angles are measured (Figure 2). The following three equations, which are completely independent, are used to adjust the error.

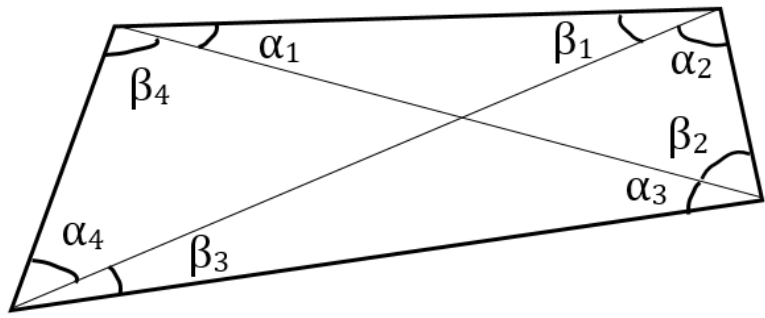

Figure 2. Figure placement and numbering

Sum of quadrilateral angles:

$$
\beta_{1}+\alpha_{2}+\beta_{2}+\alpha_{3}+\beta_{3}+\alpha_{4}+\beta_{4}=360^{\circ}
$$

Sum of opposite angles:

$$
\alpha_{1}+\beta_{1}=\alpha_{3}+\beta_{3}
$$

Sum of opposite angles:

$$
\alpha_{2}+\beta_{2}=\alpha_{4}+\beta_{4}
$$

If the above equations are not established due to operational errors, the angle adjustment operation is performed as follows.

$$
\sum \alpha_{i}+\sum \beta_{i}=360^{\circ}+\mathrm{d}
$$

Where $\mathrm{d}$ is due to errors in the observations. In this case, the adjusted angles can be calculated as bellow.

$$
\begin{aligned}
& \alpha_{i}{ }^{\prime}=\alpha_{i}-\frac{\mathrm{d}}{8} \\
& \beta_{i}{ }^{\prime}=\beta_{i}-\frac{\mathrm{d}}{8}
\end{aligned}
$$

Where $\beta_{i}{ }^{\prime}$ and $\alpha_{i}{ }^{\prime}$ are the adjusted angles for equation 11. After the adjustment, we examine equation 12 and equation 13:

$$
\alpha_{1}{ }^{\prime}+\beta_{1}^{\prime}=\alpha_{3}{ }^{\prime}+\beta_{3}{ }^{\prime}+\mathrm{e}
$$




$$
\alpha_{2}{ }^{\prime}+\beta_{2}^{\prime}=\alpha_{4}{ }^{\prime}+\beta_{4}{ }^{\prime}+f
$$

Where e and $\mathrm{f}$ are due to errors in the observations.

In order to adjust the errors in the angles to meet the latter conditions (equation 12 and equation 13) without affecting the sum of the angles (equation 11), we add $1 / 4$ of the resulting difference to the angles on the right side and subtract the angles from the left side of equation 17 and equation 18. In this case, the second adjustment form will be as follows.

$$
\begin{aligned}
& \alpha_{1}{ }^{\prime}=\alpha_{1}{ }^{\prime}-\frac{e}{4} \\
& \alpha_{3}{ }^{\prime}=\alpha_{3}{ }^{\prime}+\frac{e}{4} \\
& \beta_{1}{ }^{\prime}=\beta_{1}^{\prime}-\frac{e}{4} \\
& \beta_{3}{ }^{\prime}=\beta_{3}^{\prime}-\frac{e}{4} \\
& \alpha_{2}^{\prime \prime}=\alpha_{2}{ }^{\prime}-\frac{f}{4} \\
& \alpha_{4}^{\prime \prime}=\alpha_{4}{ }^{\prime}+\frac{f}{4} \\
& \beta_{2}^{\prime \prime}=\beta_{2}^{\prime}-\frac{f}{4} \\
& \beta_{4}^{\prime \prime}=\beta_{4}^{\prime}+\frac{f}{4}
\end{aligned}
$$

Where $\beta_{i}^{\prime \prime}$ and $\alpha_{i}^{\prime \prime}$ are the adjusted angles for equation 11 , equation 12 and equation 13. Now, considering the triangles $\mathrm{ABC}, \mathrm{BCD}, \mathrm{CDA}$, and $\mathrm{DAB}$, and using the law of sines, we can conclude that in the ideal shape we would have:

$$
\prod_{1}^{4} \sin \alpha_{i}^{\prime \prime}=\prod_{1}^{4} \sin \beta_{i}^{\prime \prime}
$$

After getting logarithms from both sides of equation 27, we have:

$$
\sum_{1}^{4} \log \sin \alpha_{i} "=\sum_{1}^{4} \log \sin \beta_{i}^{\prime \prime}
$$

Here, as well, due to the intervention of measurement errors, there is a difference between the results of the observations and the results of the theoretical relations $(\delta)$, which are expressed as follows.

$$
\delta=\sum_{1}^{4} \log \sin \beta_{i} "-\sum_{1}^{4} \log \sin \alpha_{i} "
$$

Considering:

$$
D_{i \alpha}=\log \sin \alpha_{i} "-\log \sin \alpha_{i-1} "
$$

$$
D_{i \beta}=\log \sin \beta_{i} "-\log \sin \beta_{i-1} "
$$

$\theta "$ is applied to the angles as the final adjustment:

$$
\begin{aligned}
& \theta^{\prime \prime}=\frac{\delta}{\sum_{1}^{4} D_{i \alpha}+\sum_{1}^{4} D_{i \beta}} \\
& \boldsymbol{\alpha}_{\mathbf{i}}{ }^{\prime \prime}=\boldsymbol{\alpha}_{\mathbf{i}}{ }^{\prime \prime}+\boldsymbol{\theta}^{\prime \prime} \\
& \boldsymbol{\beta}_{\mathbf{i}}{ }^{\prime \prime}=\boldsymbol{\beta}_{\mathbf{i}}{ }^{\prime \prime}-\boldsymbol{\theta}^{\prime \prime}
\end{aligned}
$$

In this case, we use the adjusted angles to calculate the lengths of the other sides, the azimuth of the sides, and finally the coordinates of the vertices of the triangle.

\subsection{Least Squares}

In this method, for every observation an equation, relating the observation to the unknown variables, is specified (Deakin, 1991). Due to measurement errors, the set of equations obtained from the observations usually do not converge to a solution and form a system of incompatible equations. It is known that different errors comprising systematic, random and operator errors, are the cause of this incompatibility. If the human operator works carefully and the device is calibrated, systematic and operator errors will not occur; therefore, we will only have random errors. Thus, we should add a random error $\left(v_{i}\right)$ to each observation. As the exact value of each $v_{i}$ is not clear, their values are considered as unknown variables. In this case the number of unknowns will be equal to $u+n$, where $n$ is the number of observations and $\mathrm{u}$ is the number of initial unknowns, and obviously the number of unknowns will be greater than the equations, so we are dealing with an under-determined system. Since under-determined systems of equations have an infinite solutions, and we want the problem to converge optimally, we have to choose the solution that minimizes the errors. For this purpose, various functions can be used as a cost function. Each of these functions are added to the system of equations, and turn the problem to an optimization problem. The Least Squares cost function is defined as bellow:

$$
\varphi=v_{1}^{2}+v_{2}^{2}+v_{3}^{2}+\ldots+v_{n}^{2}=\min
$$

Subsequently, the mathematical solution of the Least Squares method is explained.

2.5.1 Error propagation of the random errors of observations using a nonlinear parametric model: The nonlinear parametric model can generally be written as follows:

$$
l_{n \times 1}=f_{n \times u}\left(x_{u \times 1}\right)
$$

Where $x_{u \times 1}$ is the unknowns vector (here the coordinates of the vertices) with the dimension $\mathrm{u}, l_{n \times 1}$ is the observations vector, and $f_{n \times u}$ is a vector of the nonlinear functions of $x_{u \times 1}$ that transfers the unknowns to the observations. Here, the law of cosine is used as the transfer functions. Since the cause of the incompatibility in the equation 36 are the observation random errors, considering the observations errors vector $\left(v_{n \times 1}\right)$, we have:

$$
l_{n \times 1}+v_{n \times 1}=f_{n \times u}\left(x_{u \times 1}\right)
$$


In this way, the system of equations presented in equation 37 is a compatible system of equation. However, we have assumed that we do not have operator and systematic errors. Equation 37 is an under-determined system of equations, so it has infinite solutions. Considering the system of equations presented in equation 36 with the condition of the Least Squares, we have:

$$
\left\{\begin{array}{c}
l_{n \times 1}+v_{n \times 1}=f_{n \times u}\left(x_{u \times 1}\right) \\
v_{n \times 1}{ }^{\mathrm{t}} P^{-1} v_{n \times 1}=\min
\end{array}\right.
$$

Where $P$ is the observations covariance matrix. To achieve the solution of equation 38, the cost function $(\varphi=$ $\left.v_{n \times 1}{ }^{\mathrm{t}} P^{-1} v_{n \times 1}\right)$ must be minimized, which is possible by local linearization of the equations through an iterative process. Considering $x_{u \times 1}^{0}$ as an initial approximate value for the unknowns and using the Taylor series, the linear form of equation 38 is as follows:

$$
\begin{aligned}
& \left\{\begin{array}{c}
v_{n \times 1}^{i}+\delta l_{n \times 1}^{i}=A_{n \times u}^{i} \times \delta x_{u \times 1}^{i} \\
v_{n \times 1}^{i} P^{-1} v_{n \times 1}^{i}=m i n
\end{array}\right. \\
& l_{n \times 1}^{i}=f_{n \times u}\left(x_{u \times 1}^{i}\right) \\
& A_{n \times u}^{i}=\frac{\sigma f_{n \times u}}{\sigma x_{u \times 1}} \mid\left(x_{u \times 1}=x_{u \times 1}^{i}\right) \\
& \delta x_{u \times 1}^{i}=x_{u \times 1}-x_{u \times 1}^{i} \\
& \delta l_{u \times 1}^{i}=l_{u \times 1}-l_{u \times 1}^{i}
\end{aligned}
$$

The suffix $i$ at the top of each parameter indicates the desired parameter in the ith iteration. By optimizing at each step, the solution to equations 39 is obtained as follows.

$$
\begin{aligned}
& \delta x_{u \times 1}^{i}=\left(A_{n \times u}^{i-1 t} P^{-1} A_{n \times u}^{i-1}\right)^{-1} A_{n \times u}^{i-1 t} P^{-1} \delta l_{u \times 1}^{i-1} \\
& x_{u \times 1}^{i}=x_{u \times 1}^{i-1}+\delta x_{u \times 1}^{i}
\end{aligned}
$$

This repetition method is done until it converging and reaching the final answer. Thus, by using the Least Squares, the error adjustment was performed and the unknown vector was obtained.

\section{PRIMARY DATA}

In this project, simulated data were used for the analysis. The length between adjacent vertices and the angles of the network were simulated with known random errors for favorable traverse network geometries.

Statistical tests available in the Least Squares method allows us to evaluate the performance of the solution; however, this is not the case for Bowditch and Transit methods. Therefore, it is necessary to find a method to compare and evaluate the output of error propagation methods (including the Least Squares, Transit, Bowditch, and Doubly-braced Quadrilateral methods). With identical inputs, each method generates a unique series of coordinates for the network's points. Therefore, a series of data can be generated each time and according to them, the adjusted coordinates can be obtained in three methods. Here, to compare the output of different methods, the initial data was generated
2000 times and 3 methods were applied on each series of them, and the output coordinates for each method were used at each vertex to draw the error ellipse of that method.

\subsection{Generated data to compare Bowditch, Transit, and Least Squares methods}

In the first part, we are going to compare the output data (adjusted coordinates obtained from different methods) provided with Bowditch, Transit, and Least Squares methods from the input data with different accuracies. In order for the outputs of different methods to be comparable, the coordinate systems of these methods must be the same. Each two-dimensional coordinate system is characterized by four parameters ( 2 for the principal point coordinates, 1 for the scale and 1 for the orientation). Therefore, these parameters must be defined equally for the observations coordinate system. In order to consider a fixed coordinate system for the observations, we consider the first point as a control point and use a fixed observation for the first to second sight as a fixed azimuth. According to the fact that we have length observations in the network, the scale parameter is defined automatically. In order to compare the sensitivity of the methods to the relative accuracy of the observations, three observation scenarios were considered as follows.

In each network, the length of each side was considered to be 1000 meters. In the first case, the longitudinal error is considered equal to 0.1 meters. Using the error propagation law, to create an error of 0.1 meters in the specified coordinates, a 20 -second error in measuring the angles is required. Therefore, in this case, longitudinal data with a random error of 0.1 meters and angular data with a random error of 20 seconds are considered. In this case, the length and angle errors are in the same order.

In the second case, we use simulated data with a random error of 0.05 meters for the lengths and 40 seconds for the angles. In this way, the error of the lengths is halved compared to the previous state, while the error of the angles is doubled, so the mean of the errors on the vertices remain unchanged.

In the third case, we use simulated data with a random error of 0.2 meters for the lengths and 10 seconds for the angles. In this way, the error of the lengths is doubled compared to the first case, while the error of the angles is halved, so the mean of the errors on the vertices does not change.

Observational data for the three scenarios described above were tested for two shapes, including square with a side length of 1 kilometer and hexagonal with a side length of 1 kilometer, to examine the effect of network geometry on the accuracy of the methods.

\subsection{Generated data to compare Doubly-braced Quadrilateral and Least Squares methods}

In the second part, we intend to compare the output data (adjusted coordinates obtained from different methods) of the Doublybraced Quadrilateral and the Least Squares with given weights and the Least Squares with hypothetical and constant weights obtained from the input data with different accuracies with each other.

It should be noted that in this section, in order to consider a fixed coordinate system for the observations, we considered the first two points of the network as fixed points. This identifies the parameters of the transfer, scale, and orientation, therefore a fixed coordinate system is specified for the observations. 
In this case, the coordinates of the 4 vertices of the network are taken from the user and according to them, the simulated data is generated with the amount of random error specified by the user so that in each station three lines of other stations are observed along with their couplings. In this way, 6 lines are produced in each station. As mentioned earlier, only angular data are used for the Doubly-braced Quadrilateral method. Therefore, the same data should be used for the error propagation process.

In order to compare the sensitivity of the methods to the relative accuracy of the observations, three observational scenarios were considered as follows.

In the first case, we intend to produce data with a random error of 5 seconds in each station and compare the results of two methods including error propagation with the same weight for the data and Doubly-braced Quadrilateral. It should be noted that since in this case we have considered the method of the Least Squares with true weights and the Least Squares with hypothetical weights, both with a constant weight of 5 seconds, the resulting error ellipses related to them overlaps.

In the second case, we want to produce data with different random errors in each station and compare the results obtained from the three methods of the true-weighted Least Squares, Least Squares with constant and hypothetical weights for the data and Doubly-braced Quadrilaterals. In this case, for the each observed angles, a different random error is considered, in a way that the first (A), second (B), third (C) and fourth (D) vertices have random errors of $10,8,4$, and 1 seconds, respectively. Therefore, the average of these errors is equal to 5 seconds. In the trueweighted Least Squares method, the true observation errors were used to derive the observations weights; however, for the Least Squares method with hypothetical constant weights, the hypothetical constant weight corresponding to the random error of 5 seconds is considered for all observations in the solution method.

The observational data for the two scenarios described above were tested for three shapes of squares with a side length of $1 \mathrm{~km}$, parallelograms (Figure 3), and irregular quadrilaterals (Figure 4) to examine the impact of the geometry of the network on the accuracy of various methods.

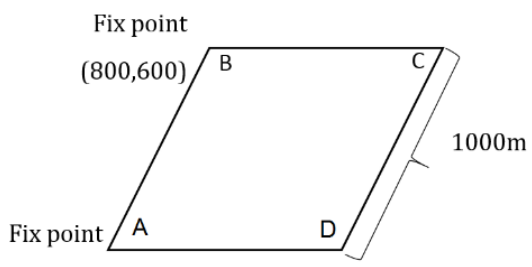

$(0,0)$

Figure 3. The parallelograms specification.

Fix point

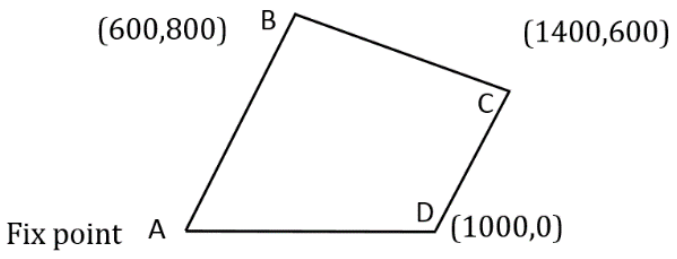

$(0,0)$

Figure 4. The irregular quadrilateral specification.

\section{DISCUSSION AND RESULTS}

In this section, we investigate the results of comparing different adjustment methods on the data and different geometries. In the first part, we examine the solutions of the adjustment methods, including Transit, Bowditch, and the Least Squares methods under the previously described scenarios. Then, in the last section, the Doubly-braced Quadrilateral adjustment method and the Least Squares are evaluated under the scenarios introduced above.

\subsection{Investigating Bowditch, Transit and Least Squares methods}

The following results are evident in Figure 5 and Figure 6, which was observed in both geometries. In Transit method, by deviating from the first point in the direction of the adjustment, the error ellipses grow larger, hence the accuracy of the coordinates at the vertices depends directly on the direction of the work being done. In the Least Squares and Bowditch methods, the accuracy decreases by moving away from the control point in any direction so that in the adjacent points to the control point, the ellipses are similar and their dimensions are smaller than the error ellipses of the farther points, in fact, the more we get farther from the control points, the more the ellipses grow. The Bowditch method, similar to the Least Squares method, considers the relative accuracy of the observations in its calculations. This means, for example, that by increasing the accuracy of the length over the accuracy of the angle, the error ellipses of this method, alike the error ellipses of the Least Squares method, become more elongated in the direction of the angular error. In the first vertex, due to the high precision angle observation (azimuth along the first line), unlike the Bowditch method, the ellipses of the Least Squares and Transit methods have error ellipses in the observed length direction, so these two methods apply the accuracy of this observation to the calculations. By increasing the accuracy of the angular observations over the length observations, the Bowditch method has got closer to the Least Squares at some network vertices and gives better accuracy. As the accuracy of the original data is diverged from the equilibrium state (i.e., the angular accuracy is equal to the longitudinal accuracy, the state in Figure $5 \mathrm{~b}$ and Figure $6 \mathrm{~b}$ ), the error ellipses of all three methods become larger. Table 1 (Appendix) showed the size of the large and the small diameters of the ellipses for a regular hexagon (Figure $6 \mathrm{~b}$ ). It can be seen from Table 1 that the size of the error ellipse was at the same order with the measurements error when the accuracy of angular and longitudinal observations were of the same order. While the equilibrium between the longitudinal and angular observations error was disturbed, the error ellipse became larger.

\subsection{Doubly-braced Quadrilateral method results}

Investigation the results on the data, when the input data were of equal accuracy, showed that the Least Squares method with constant weight and the Doubly-braced Quadrilateral method give similar accuracies.

But as shown in Figure 7, when the input data had different accuracies, the Least Squares with constant weight and the Doubly-braced Quadrilateral methods had similar accuracies, while the true-weighted Least Squares method gave a better accuracy than the previous two methods.

In quadrilateral shapes independent from the accuracy of the data and the method of the calculation, the accuracy of the data obtained for vertices with an angle greater than 90 degrees is 
better than vertices with an angle less than 90 degrees; however, the angle should be sufficiently far from 180 degrees.

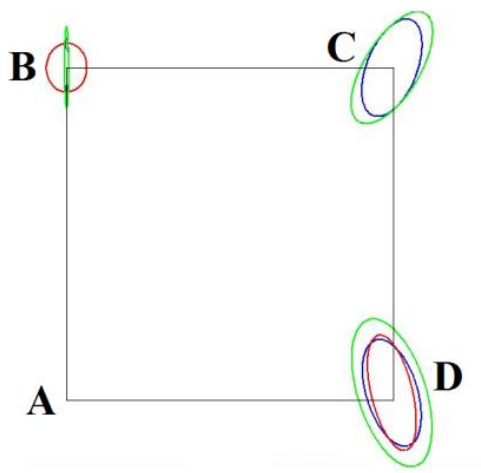

(a)

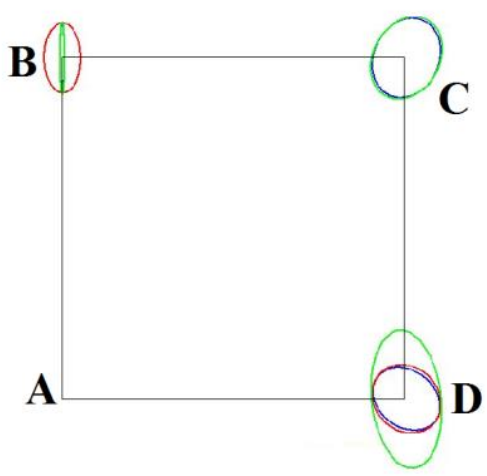

(b)

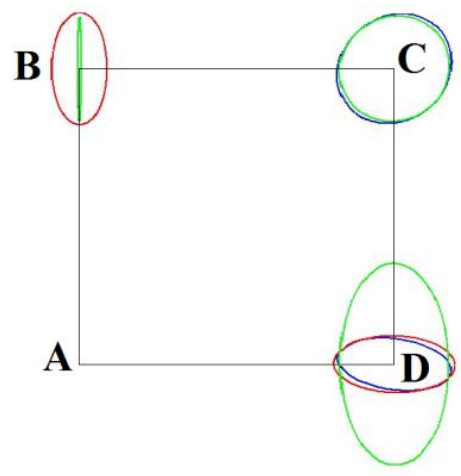

(c)

Figure 5. Error ellipses for results obtained from three adjustment methods of Transit (shown in green), Bowditch (shown in red) and the Least Squares (shown in blue) for simulated data for a square grid with a side length of one kilometer. In case a, an observation error of $5 \mathrm{~cm}$ for the lengths and 40 seconds for the angles, in case $b$, an observation error of $10 \mathrm{~cm}$ for the lengths and $20 \mathrm{~s}$ for the angles, and in case $\mathrm{c}$, an observation error of $20 \mathrm{~cm}$ for the lengths and 10 s for the angles was considered. Error ellipses are magnified by 1000 times.

\section{CONCLUSIONS}

As can be seen from the results section, the Least Squares method in most cases gave the best solution among the other methods. When performing a traverse, the Bowditch method in many

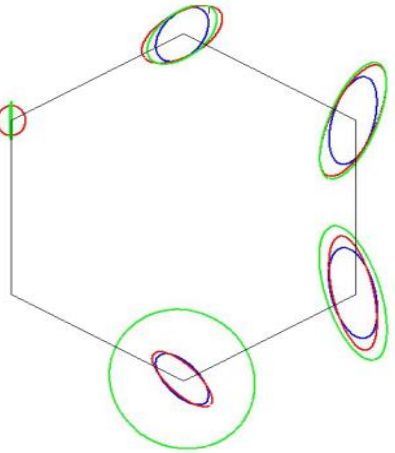

(a)

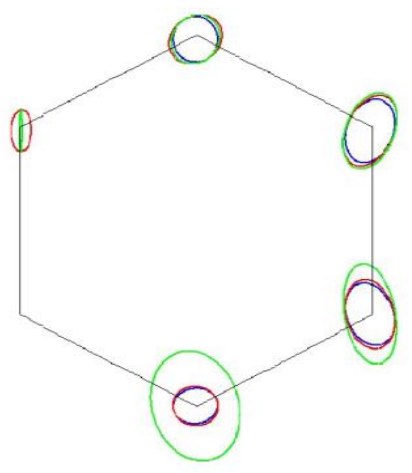

(b)

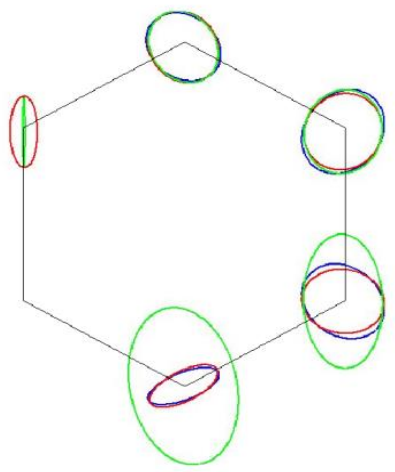

(c)

Figure 6. Error ellipses for results obtained from three adjustment methods of Transit (shown in green), Bowditch (shown in red) and the Least Squares (shown in blue) for simulated data for a regular hexagonal grid with side lengths of one kilometer. In case a, an observation error of $5 \mathrm{~cm}$ for the lengths and 40s for the angles, in case $b$, an observation error of $10 \mathrm{~cm}$ for the lengths and 20s for the angles, and in case $\mathrm{c}$, an observation error of $20 \mathrm{~cm}$ for the lengths and $10 \mathrm{~s}$ for the angles was considered. Error ellipses are magnified by 1000 times.

vertices gave a similar answer to the Least Squares method, which in fact indicates a good propagation of the observation errors between the observations in this method. In addition, in the Doubly-braced Quadrilateral method, the results were always the same with the Least Squares with constant weight, which also results from the proper error propagation in this method. 
However, when the accuracy of the observations differed, i.e. the weight and the importance of the observations varied, the Least Squares method, unlike the Doubly-braced Quadrilateral method, had the potential to prioritize the observations with higher accuracies in the calculations. In general, the compatibility of these geometric methods with the Least Squares method indicates the appropriate distribution of the errors in these methods.

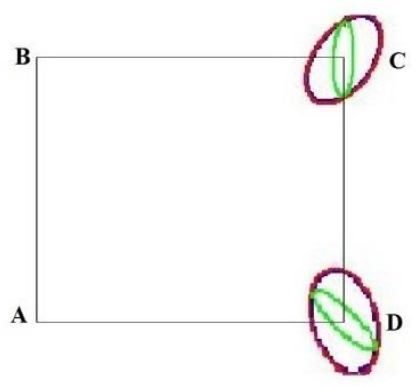

(a)

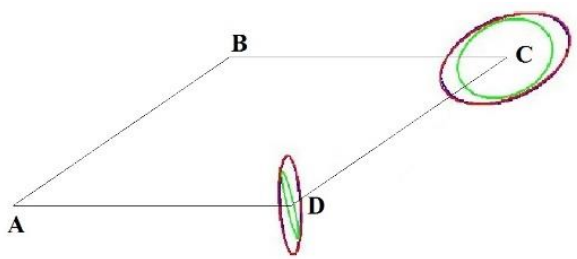

(b)

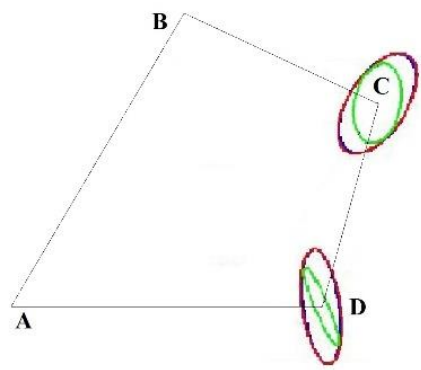

(c)

Figure 7. Error ellipses for results obtained from three adjustment methods of the true-weighted Least Squares (shown in green), the Least Squares with a constant and hypothetical weight of 5 seconds (shown in red) and the Doubly-braced Quadrilateral (shown in blue) for simulated data for (a) square (b) parallelogram (c) irregular quadrilateral specified in section 3.2. The actual accuracy of the observations is considered with a random error of 10 seconds for the first vertex, 8 seconds for the second vertex, 4 seconds for the third vertex, and 1 second for the fourth vertex of the traverse. Error ellipses are magnified by 1000 times.

\section{REFERENCES}

Chrzanowski, A. and Konecny, G., 1965. Theoretical comparison of triangulation, trilateration and traversing. The Canadian Surveyor, 19(4), pp.353-366.

Deakin, R.E., 1991. A review of least squares theory applied to traverse adjustment. Australian surveyor, 36(3), pp.245-253.
GTM, 1938. BOWDITCH TRAVERSE ADJUSTMENT AND A MODIFICATION. Empire Survey Review, 4(29), pp.417-425. Gutti, M.B. and Musa, A.A., 2019. SETTING OUT OF CURVES AND ROUTE SURVEYING FOR A ROAD.

Kimmick, E.J., 1949. Analysis of the transit traverse survey method of the United States Department of Interior, Geological Survey.

Thornton-Smith, G.J., 1964. Adjustment of Measured Angles and Measured Lengths in a Doubly-Braced Quadrilateral. Survey Review, 17(131), pp.236-240.

\section{APPENDIX}

\begin{tabular}{|c|c|c|c|c|c|c|}
\hline \multirow{2}{*}{ Vertices } & \multicolumn{2}{|c|}{ B } & \multicolumn{2}{|c|}{$\mathrm{C}$} & \multicolumn{2}{|c|}{$\mathrm{D}$} \\
\hline & a & $\mathrm{b}$ & $\mathrm{a}$ & $\mathrm{b}$ & a & $\mathrm{b}$ \\
\hline Proration & 0.092 & 0.005 & 0.102 & 0.091 & 0.143 & 0.095 \\
\hline Bowditch & 0.092 & 0.041 & 0.118 & 0.099 & 0.164 & 0.096 \\
\hline Transit & 0.094 & 0.005 & 0.112 & 0.096 & 0.175 & 0.098 \\
\hline Vertices & \multicolumn{2}{|c|}{$E$} & \multicolumn{2}{|c|}{$\mathrm{F}$} & & \\
\hline Methods & a & $\mathrm{b}$ & $\mathrm{a}$ & $\mathrm{b}$ & & \\
\hline Proration & 0.143 & 0.094 & 0.092 & 0.077 & & \\
\hline Bowditch & 0.156 & 0.096 & 0.093 & 0.085 & & \\
\hline Transit & 0.226 & 0.102 & 0.248 & 0.177 & & \\
\hline
\end{tabular}

Table 1. The length of the larger diameter (b) and the smaller diameter (a) of the error ellipses in meters obtained from the analysis of the regular hexagonal results with an observation error of 10 centimeters for the lengths and 20 seconds for the angles. 